\title{
Acknowledgement to BJD 2018 reviewers
}

\section{We are grateful to the following people who acted as reviewers for articles submitted to the British Journal of Diabetes during 2018.}

Dr James Burton, Leicester General Hospital

Dr Peter Carey, Sunderland Royal Hospital

Dr Ali Chakera, Royal Sussex County Hospital

Dr Kelly Cheer, Wythenshawe Hospital

Dr Pratik Choudhary, Kings College Hospital

Professor Deborah Christie, University College London Hospitals

Dr MarkDavies, Belfast Health \& Social Care Trust

Dr Caroline Day, Halesowen

Dr Ketan Dhatariya, Norfolk \& Norwich University Hospital

Dr Jackie Elliott, Sheffield Teaching Hospitals

Dr Michael Feher, Chelsea and Westminster Hospital

Dr Alison Gallagher, University Hospitals of Leiceser

Dr Rob Gregory, University Hospitals of Leicester NHS Trust

Dr Roselle Herring, Royal Surrey County Hospital

Dr Rajesh Jogia, University Hospitals of Leicester NHS Trust

Dr Dhanya Kalathil, Royal Liverpool and Broadgreen University Hospitals NHS Trust

Dr Anne Kilvert, Northampton General Hospital
Dr Chantal Kong, West Hertfordshire Hospitals NHS Trust

Dr Lalantha Leelarathna, Manchester Diabetes Centre, Manchester Royal Infirmary

Professor Neil Munro, University of Surrey

Dr Vakkat Muraleedharan, King's Mill Hopital

Dr Dinesh Nagi, Pinderfields Hospital

Dr Parth Narendran, The Queen Elizabeth Hospital

Dr Rahul Nayar, Sunderland Royal Hospital

Dr Emmanuel Kofi Obuobie, Royal Gwent Hospital

Dr Nikolaos Papanas, Greece

Professor Vinod Patel, George Elliot Hospital

Dr Faizanur Rahman, University Hospitals of Leicester NHS Trust

Professor SatyanRajbhandari, Lancashire Teaching Hospital

Dr Sud Ramachandran, Good Hope Hospital

Dr Victoria Ruszala, North Bristol NHS Trust

Dr Jonathan Schofield, Manchester Royal Infirmary

Dr Chris Walton, Hull Royal Infirmary

Dr Jonathan Webber, University Hospitals Birmingham

Dr Emma Wilmot, Derby Teaching Hospitals

Dr Peter Winocour, ENHIDE 